\title{
Clinical applications of cortisol measurements in hair
}

\author{
Vincent L Wester ${ }^{\dagger}$ and Elisabeth F C van Rossum ${ }^{*}$ \\ Division of Endocrinology, Department of Internal Medicine, Erasmus MC, University Medical Center Rotterdam, \\ PO Box 2040, 3000 CA Rotterdam, The Netherlands \\ ${ }^{+} \mathrm{V}$ L Wester is now at Room Ee514, Erasmus MC, PO Box 2040, 3000 CA Rotterdam, The Netherlands \\ ${ }^{\ddagger} \mathrm{E}$ F C van Rossum is now at Room D428, Erasmus MC, PO Box 2040, 3000 CA Rotterdam, The Netherlands
}

Correspondence

should be addressed

to E F C van Rossum

Email

e.vanrossum@erasmusmc.nl

\begin{abstract}
Cortisol measurements in blood, saliva and urine are frequently used to examine the hypothalamus-pituitary-adrenal (HPA) axis in clinical practice and in research. However, cortisol levels are subject to variations due to acute stress, the diurnal rhythm and pulsatile secretion. Cortisol measurements in body fluids are not always a reflection of long-term cortisol exposure. The analysis of cortisol in scalp hair is a relatively novel method to measure cumulative cortisol exposure over months up to years. Over the past years, hair cortisol concentrations (HCC) have been examined in association with a large number of somatic and mental health conditions. HCC can be used to evaluate disturbances of the HPA axis, including Cushing's syndrome, and to evaluate hydrocortisone treatment. Using HCC, retrospective timelines of cortisol exposure can be created which can be of value in diagnosing cyclic hypercortisolism. HCC have also been shown to increase with psychological stressors, including major life events, as well as physical stressors, such as endurance exercise and shift work. Initial studies show that HCC may be increased in depression, but decreased in general anxiety disorder. In posttraumatic stress disorder, changes in HCC seem to be dependent on the type of traumatic experience and the time since traumatization. Increased hair cortisol is consistently linked to obesity, metabolic syndrome and cardiovascular disease. Potentially, HCC could form a future marker for cardiovascular risk stratification, as well as serve as a treatment target.
\end{abstract}

\section{Introduction}

The glucocorticoid hormone cortisol is produced by the hypothalamus-pituitary-adrenal (HPA) axis, and has effects on virtually all tissues and processes in the human body (1). Biochemical evaluation of cortisol, including dynamic testing, is the cornerstone of diagnosing hypo- and hypercortisolism (2). Besides these obvious endocrine disorders, subtle variations in cortisol exposure and sensitivity have been extensively studied in relation to a wide range of health and disease characteristics, including aspects of metabolism, inflammation, cardiovascular disease and behavior (1).

Systemic cortisol levels are highly variable, which is due to the diurnal rhythm, acute stress and pulsatile secretion (3). Measurements of cortisol in blood, saliva

Invited Author's profile

E F C van Rossum MD PhD is Internist-Endocrinologist, Associate Professor, member of the medical staff of the Department of Internal Medicine, and head of the Outpatient Clinic of Endocrinology at the Erasmus University Medical Center, Rotterdam, The Netherlands. At present, she is also head of the adult Division of the Obesity Center CGG, an academic center for diagnosis of underlying causes of obesity and innovative treatment strategies. Her research is focused on the genetics of individual glucocorticoid sensitivity and the role of long-term cortisol exposure in health and disease (obesity, cardiometabolic diseases and psychiatric disorders).

(c) 2015 European Society of Endocrinology Printed in Great Britain Published by Bioscientifica Ltd.
This article is adapted from work presented at IMPROCUSH-1, 12-14 October 2014. The meeting was supported by the European Science Foundation, Deutsche Forschungsgemeinschaft, Carl Friedrich von Siemens Stiftung, European Neuroendo-

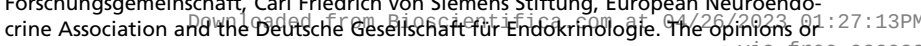
views expressed in this article are those of the authors, and do not necessarily reflecte access

the opinions or recommendations of the supporters of the symposium. 
and urine only represent time-point or short-term cortisol exposure, which is limiting the representation of longterm circulating cortisol levels. Furthermore, these tests either rely heavily on patient adherence to collection instructions, or are perceived as invasive (2).

Scalp hair analysis has been used for decades to measure exposure to environmental toxins and drugs. Hair grows at a relatively constant rate of $1 \mathrm{~cm} /$ month. Several substances are retained in the hair strands, therefore, hair can serve as a matrix to measure longterm exposure to a wide variety of toxins and hormones. Furthermore, by dividing hair samples in different segments and analyzing them separately, retrospective timelines of exposure can be created (4).

In the past years, several laboratories have expanded hair analysis to measure long-term levels of cortisol and other steroid hormones $(5,6,7,8,9,10)$. This has allowed researchers to investigate cortisol exposure over much longer periods of time (months to years) than previously possible with samples of blood, saliva or urine. Consequently, a large number of cross-sectional studies have been conducted and examined the associations between hair cortisol concentrations (HCC) and a wide range of somatic and mental health measures $(11,12)$. In this review, we will highlight current and potential future applications of hair cortisol measurements, embed this relatively novel method in the context of other available tests, and discuss potential confounders and future directions of HCC measurements.

\section{Hair sample collection and analysis}

Scalp hair collection is straightforward and easily performed in an outpatient setting (Fig. 1). In accordance with guidelines published by the Society of Hair Testing, the hair sample is collected from the posterior vertex (4). Scissors are used to cut a lock of hair as thick as a pencil, as close to the scalp as possible. Depending on the research question, hair segments of one, up to multiple centimeters of length are used for analysis. Samples are weighed, and in most published methods washed. Steroids are extracted in methanol overnight and are then processed further depending on the type of analysis used. The extracted cortisol can be measured using an immunoassay or liquid chromatography tandem-mass spectrometry (LC-MS/MS) $(5,6,7,8,9,10)$.

Recently, a direct comparison between hair cortisol analysis methods was published. Four different laboratories divided hair samples of the same persons and measured them using their different methods, comparing four immunoassay methods and two LC-MS/MS methods. Correlations between the different methods were high, with $r^{2}$ values ranging between 0.88 and 0.98 (13).

\section{Applications in clinical practice and research}

Thus far, HCC have been associated with a wide range of clinical outcomes, the results of which are summarized in Table 1. The published clinical outcomes can be divided in several categories: Cushing's syndrome (CS), hydrocortisone replacement therapy monitoring, cardiometabolic status, psychopathology and either physical or psychological forms of chronic stress. Other factors that can influence HCC, such as sex, age and hair-related factors, are discussed in 'Factors affecting HCC' section.
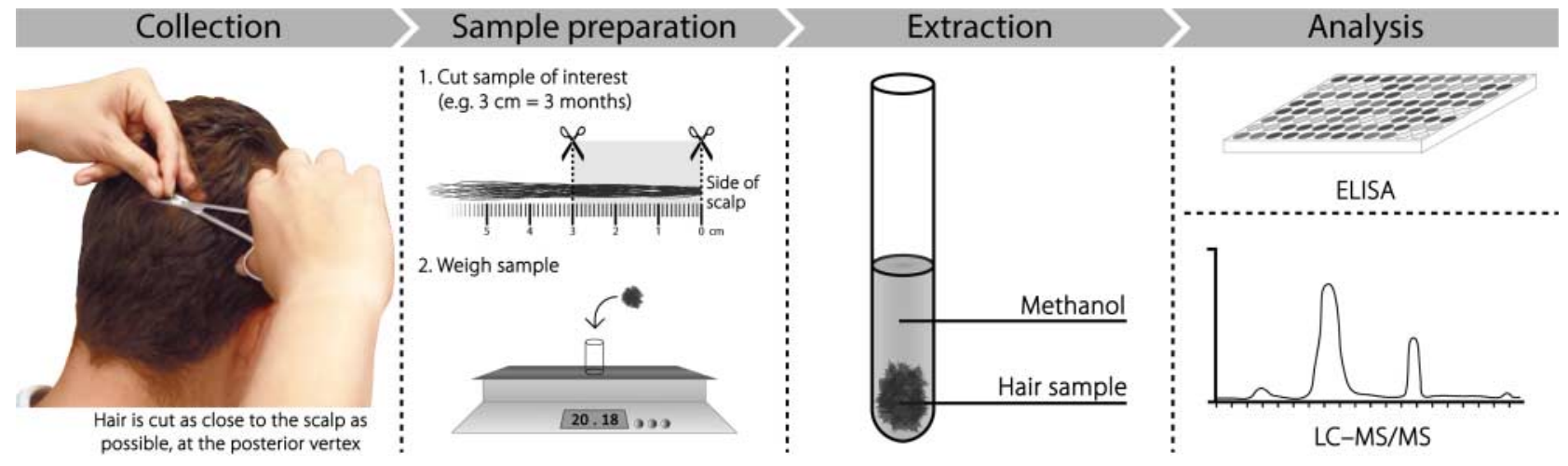

Figure 1

Overview of hair sample collection, work-up and analysis. ELISA, enzyme-linked immunosorbent assay LC-MS/MS, liquid chromatography-tandem mass spectrometry. 
Table 1 Clinical and situational factors associated with higher or lower hair cortisol levels.

\begin{tabular}{|c|c|c|}
\hline & Increased hair cortisol & Decreased hair cortisol \\
\hline \multicolumn{3}{|l|}{ Somatic health factors } \\
\hline & Cushing's syndrome & Childhood asthma with inhalation glucocorticoids \\
\hline & Hydrocortisone use & \\
\hline & Obesity & \\
\hline & Metabolic syndrome & \\
\hline & Diabetes mellitus & \\
\hline & Cardiovascular disease & \\
\hline & Heart failure severity & \\
\hline & Recent myocardial infarction & \\
\hline \multicolumn{3}{|l|}{ Chronic and acute stressors } \\
\hline & Intensive aerobic exercise & Traumatic experience \\
\hline & Trauma & \\
\hline & Life events & \\
\hline & Unemployment & \\
\hline & Shift work & \\
\hline & Severe chronic pain & \\
\hline \multicolumn{3}{|l|}{ Psychopathology } \\
\hline & PTSD $^{a}$ & PTSD $^{a}$ \\
\hline & Major depressive disorder & Generalized anxiety disorder \\
\hline & Bipolar disorder, late onset & Panic disorder \\
\hline
\end{tabular}

aposttraumatic stress disorder (PTSD) has been associated with both increased and decreased hair cortisol concentrations (depending on the type of traumatic event, characteristics of the patient sample examined, and the timespan between the trauma and assessment), when compared to controls.

\section{Cushing's syndrome}

We and others have previously reported increased HCC in patients with endogenous CS $(14,15,16)$. In a sample of 14 CS patients and 96 healthy controls, we found a sensitivity of $86 \%$ and a specificity of $98 \%$ for CS, which is comparable to the diagnostic accuracy of urinary free cortisol (UFC), late night salivary cortisol (LNSC), and the low-dose dexamethasone suppression test (DST) (14). Since our study only included 14 patients with CS and was not designed to compare HCC with other tests, future studies have to be awaited to in order to determine the value of HCC in diagnosis and surveillance for recurrence of CS. Cortisol secretion is known to be highly variable in CS, with a majority of patients showing at least one normal UFC or LNSC in repeated testing (17). By providing information about cortisol exposure over a much longer timeframe, measurement of HCC may help to overcome this issue. Furthermore, unlike current first-line screening tests for CS, hair sample collection is not limited by patient adherence to collection instructions (as with UFC of LNSC) or to medication intake (as with the low-dose DST).

One of the main advantages of hair measurements is the possibility to create retrospective timelines of cortisol exposure, months up to years back in time, depending of the length of the hairs. Furthermore, depending on the length of the hair, HCC may be used to indicate the time of onset of the disease. We and others have described cases of CS in which the timelines of HCC corresponded with disease course $(14,15,16)$, and showed that creating timelines can be especially helpful in cases of suspected cyclic CS. In cyclic CS, duration of episodes of hypercortisolism and normocortisolism are unpredictable, varying form several days to months or years. Cyclic CS can therefore be very challenging to diagnose, because cortisol production may appear normal in biochemical evaluation (18). By expanding the time window in which cortisol is measured, and the addition of retrospective timelines, which can be individualized based on the clinical symptoms of the patient, HCC have the potential to improve the detection of cyclic CS cases considerably.

\section{Hydrocortisone replacement therapy monitoring}

Hydrocortisone prescribed for adrenal insufficiency (AI) is chemically identical to cortisol. Therefore HCC may be used to assess long-term systemic levels of administered hydrocortisone in AI patients. Several published studies have investigated HCC in patients treated with hydrocortisone for AI. The studies included 132 adults with AI, 93 adults with AI, 54 children with AI and 15 adults who were treated with the adrenolytic drug mitotane for adrenocortical carcinoma (19, 20, 21, 22). All studies reported higher mean HCC in hydrocortisone-treated patients compared to healthy individuals or non-AI controls. In the two studies performed in adult AI patients, 
HCC correlated significantly with hydrocortisone dose $(19,20)$, while in the other two studies, HCC correlated significantly with BMI but not with hydrocortisone dose $(21,22)$. In the adrenocortical carcinoma patients, this can be explained by mitotane treatment, which alters the metabolism of hydrocortisone. These results indicate that HCC may be used to identify overtreatment with hydrocortisone. Combining HCC with clinical symptoms of hydrocortisone overtreatment may therefore improve refinement of individualized hydrocortisone substitution therapy. However, glucocorticoid action is affected by a large number of factors. These include intra-individual differences in hydrocortisone absorption and breakdown which are likely reflected in hair levels, but also differences in glucocorticoid sensitivity which alter cortisol effects at the tissue level (1).

Next to hydrocortisone replacement therapy, other glucocorticoids are commonly used to treat a wide variety of diseases (e.g. inflammatory and autoimmune diseases, malignancies). Glucocorticoid use may suppress the HPA axis, but since hydrocortisone is identical to cortisol, HCC cannot be used to distinguish between exogenous and endogenous cortisol. However, other synthetic glucocorticoids may be distinguished from cortisol by HCC measurements. One recent study found lower HCC in children who used inhaled glucocorticoids for asthma, providing preliminary evidence that HCC might be used to detect HPA axis suppression due to exogenous glucocorticoid use (23).

\section{Cardiometabolic status}

Excessive glucocorticoid exposure is associated with increases in cardiometabolic risk factors such as obesity, dyslipidemia, glucose intolerance and hypertension (24). However, these observations come from patients with an overt excess of glucocorticoids due to glucocorticoid therapy or endogenous hypercortisolism. Studies using cortisol levels derived from body fluids could not fully unravel the relationship between variations in cortisol exposure within the physiologic range and cardiovascular risk (24). Cross-sectional studies using hair analysis have shown that higher HCC are associated with an adverse cardiovascular risk profile. We have found that in a population of 283 elderly, individuals in the highest quartile of HCC were 2.7 times more likely to suffer from cardiovascular disease, whereas no relationship with noncardiovascular chronic disease (e.g. cancer and obstructive lung disease) was found. Furthermore, HCC were positively associated with presence of type 2 diabetes mellitus (25), a finding which was confirmed in another population-based elderly cohort (26). HCC have been found to be increased in patients with a recent myocardial infarction (27). In a large cohort of 1258 middle-aged aviation workers, Stalder et al. (28) found higher hair cortisol and cortisone levels associated with increased presence of the metabolic syndrome, showing that the relationship between HCC and adverse cardiometabolic status is already present earlier in life.

The association between cortisol levels and obesity has been a subject of research for a long time. Results from past studies are conflicting. Urinary cortisol has been reported increased in obese individuals compared to lean individuals, but this increase is generally not reflected in serum or salivary cortisol levels (24). We found that in both adult and pediatric obese patients, HCC are increased compared to non-obese healthy controls $(29,30)$. Furthermore, HCC positively correlates with BMI in multiple other studies (21, 28, 31, 32, 33, 34). Together, these results strongly suggest that increased long-term cortisol exposure is associated with increased adiposity. An increased longterm cortisol exposure may possibly be responsible for part of the increased cardiovascular disease risk in obese individuals. However, no causal inferences can be drawn from these studies. Long-term cortisol exposure may not only be important in developing an increased cardiovascular disease risk profile, but may also have modulating effects in cardiac disease. This is illustrated by the finding that higher HCC have been associated with worse functional status in heart failure (35), and with worse subjective physical health in structural heart disease (32).

\section{Psychopathology}

Cortisol is thought to play an important role in psychopathology, in particular in mood and anxiety disorders. Depressed individuals have been found to take more time to return to baseline cortisol levels in response to a psychological stressor compared to non-depressed controls (36). Furthermore, patients with current and remitted depression appear to have an increased cortisol awakening response (37). An increased HPA axis reactivity in patients with depression may be caused by a relative resistance to cortisol, as evidenced by a blunted response to the DST in depressive patients (38). One relatively small study, involving 23 patients with major depressive disorder and 64 matched controls, found an increase in HCC in depressed patients using antidepressant medication (39). In patients with bipolar disorder we also identified a subgroup with an older age of onset having increased 
HCC (40). These results indicate that an altered HPA axis activity in mood disorders may result in increased longterm cumulative cortisol levels.

Cortisol is also thought to play a role in anxiety disorders. In a small sample of patients with generalized anxiety disorder (GAD), decreased HCC have been reported. This is in contrast to results obtained from short-term or acutely circulation concentrations, which have been reported to be increased in GAD (41). In a population of patients with bipolar disorder, we have found that those who had a comorbid panic disorder had decreased HCC, while salivary cortisol levels were unaffected by panic disorder in this study (40). These discordances could be explained by the fact that a research setting can form an acute stressor, which could increase acutely circulating cortisol levels, but not affect cortisol levels in scalp hair. Furthermore, these examples demonstrate clearly that hair cortisol, representing long-term cumulative exposure, can offer valuable new information in addition to the time point measurements, which are more suitable to obtain insight in acute changes.

Traumatic experience and posttraumatic stress disorder (PTSD) have also been associated with alterations in HPA axis function. A traumatic experience will result in acutely increased cortisol levels due to an acute stress reaction. Observational studies suggest that after the traumatic event individuals who develop PTSD have lower circulating cortisol levels. However, a recent metaanalysis failed to show an overall difference in cortisol levels in urine, blood or saliva between people with PTSD $(n=828)$ and controls $(n=800)$ (42). Subgroup analysis in this study revealed lower short-term or time point cortisol levels in female PTSD patients, and in PTSD subjects who were traumatized due to physical or sexual abuse, but not in those who were traumatized due to war. Furthermore, a decrease was only observed in studies in which PTSD subjects were compared to control subjects who were not exposed to trauma, but not when compared to traumatized controls without PTSD (42). This indicates that trauma exposure, rather than the subsequent development of PTSD is responsible for a decrease in cortisol. Findings concerning HCC and PTSD are largely in line with this. Steudte et al. (43) reported that both PTSD and non-PTSD traumatized controls both have lower HCC when compared to nontraumatized controls. However, Luo et al. described that in adolescent females who experienced an earthquake, HCC was lower in the 32 subjects who developed PTSD, than in 32 persons who did not. This relative decrease was seen in hair segments corresponding to the second through seventh month after the earthquake, but not before that time (44), indicating that the decrease in cortisol levels only occurs after the traumatic event. Moreover, in another study involving 202 women who survived the same earthquake, HCC levels were not associated with PTSD symptoms 4.5 years after the event (45). Contrary to decreases that are observed in most studies, in 27 traumatized individuals from a civil war area in Uganda, those who developed PTSD had significantly higher HCC (46). In conclusion, the association between long-term cortisol exposure and PTSD appears to be dependent on the type of traumatic event, characteristics of the patient sample examined, and, importantly, the timespan between the trauma and assessment.

\section{Chronic stress}

Both physical and psychological stress can induce a biological stress response in the human body, including a hyperactivation of the HPA axis. Much of what is known about HPA axis responses to stressors is derived from measurements of cortisol in saliva. In particular, salivary cortisol is used to investigate changes in the cortisol awakening response, and the response to experimentally induced stress, such as in the Trier Social Stress test (47). As mentioned before, HCC can measure cortisol levels over extended periods of time, and unlike salivary and serum measurements, is not affected by the influence of acute stress or time-of-day at the moment of sampling. Therefore, it is a highly attractive method to investigate the association between real-life stressors and long-term cortisol exposure.

Examples of physical stressors are severe diseases, extreme exercise and starvation. Another common physical, and maybe also partially psychological stressor is disruption of the circadian rhythm (11). We have reported an increase in HCC in shift workers, compared to day workers (31). This is an important finding, because shift work is associated with metabolic syndrome components, and cortisol may be in part responsible for this association. Interestingly, in this study BMI correlated positively with HCC (31). Other examples of physical stress, associated with elevated HCC are endurance exercise (48) and chronic pain (49).

The relationship between experienced psychological stress and the HPA axis is complex. Conflicting results have been reported in the association between perceived stress, and HCC. Both increased (50) and decreased HCC (51) have been associated with higher perceived stress, while other studies have failed to find a relationship 
altogether $(33,52)$. This heterogeneity could be a consequence of the diverse composition of study samples, which range from coronary artery disease patients (52) to college students (51). However, published research supports an association between recently experienced (negative) life events, such as loss of a loved one or an important exam, and increased HCC or hair cortisone in college students (51), elementary school girls (53), patients with bipolar disorder (54) and crack cocaine users (55). Other psychological stressors which have been associated with increased HCC are school entry in children (56), giving care to a relative with dementia (57), promotion prospects (58) and unemployment (59).

\section{Factors affecting HCC}

Apart from the clinical states and stressors that we discussed in this review, several other factors can potentially affect HCC. In the largest published studies, HCC increased with age $(26,28)$. Also, children have been found to have significantly lower HCC than adults (60). Several published studies report higher HCC in males than in females $(25,26,61)$, but in other studies no sex difference was observed $(9,28,33)$. In two studies carried out in older adults, men had higher hair cortisol, indicating that the sex difference in HCC becomes more pronounced later in life $(25,26)$. The associations between ethnicity and HCC have largely been unexplored, and need further study.

Multiple studies have confirmed a hair growth rate that closely $\sim 10 \mathrm{~mm} /$ month (62). Slight differences in hair growth rate between ethnic groups have been reported, with African hair growing more slowly than Caucasian hair, which in turn grows more slowly than East Asian hair (63). Small variations in growth rate are not expected have a large impact on studies using single measurements of long-term cortisol, but should be considered when creating longer retrospective timelines.

Hair treatments such as hair dyeing, permanent curling or straightening have been reported to decrease $\operatorname{HCC}(9,28,64)$, although other studies have not found this $(6,33,48,52,60,65)$. In the largest published HCC study thus far, in which hair glucocorticoids were measured in 1258 individuals using LC-MS/MS, cortisol and cortisone were lower in hair which was colored, permanently curled or straightened. Furthermore, the amount of cortisone decreased with higher hair washing frequency in this study (28). Another hair-specific limitation is the phenomenon of wash-out, which means that hair cortisol is lower more distally in the hair, for instance due to wear and tear, subsequent hair washings or exposure to ultraviolet light. We have previously found that HCC was stable in subsequent hair segments in 28 women over a hair length of $18 \mathrm{~cm}$ measured using an immunoassay (9), but we did observe a decrease in cortisol levels along the hair shaft in our LC-MS/MS based method (10). Others have described a wash-out effect in HCC as well $(6,66)$. Therefore, wash-out of hair steroids is an important consideration, especially in studies involving retrospective timelines using segmental hair analysis.

It is not yet known how cortisol enters the hair shaft. It is assumed that cortisol enters the hair shaft through passive diffusion, and that HCC therefore represent cumulative free circulating cortisol levels. This is further supported by the circumstantial evidence that oral contraception, which is known to increase total cortisol levels due to the effect of estrogens on corticosteroid binding globulin levels (2), does not seem to have a major influence on HCC $(33,67)$. However, active transport of steroids from the blood stream to the hair follicle cannot be excluded. Apart from incorporation from blood, sebum and sweat could also contribute to HCC. Cortisol has been demonstrated in human sweat, and it has been suggested that increased sweating may increase HCC, which may acutely influence HCC and therefore undermine the validity of HCC as a marker of long-term cortisol exposure (68). However, Stalder et al. recently showed that two sweat-inducing interventions (exercise and sauna bathing) do not seem to acutely influence HCC (69).

Multiple studies have measured both cortisol and cortisone in scalp hair using LC-MS/MS $(10,28)$. Interestingly, in a study by Stalder et al. (28) hair cortisone levels correlated stronger with cardiovascular risk factors than hair cortisol did. This suggests that hair cortisone levels offer valuable information about systemic glucocorticoid exposure. Furthermore, determination of both cortisol and cortisol in hair may provide information about the activity of $11 \beta$-hydroxysteroid dehydrogenase (11 $\beta$-HSD) enzymes, which convert the active cortisol into cortisone and vice versa. However, 11 $\beta$-HSD enzymes are expressed in skin as well (70), and may therefore impact the ratio between cortisol and cortisol in hair locally, potentially limiting the value of this ratio as a marker of systemic $11 \beta$-HSD activity.

Medication use is known to influence different types of cortisol measurements (2), but until now this does not seem to be a major limiting factor in HCC measurements. It is important however, to consider the use of topical 
steroids which may contaminate hair samples, and falsely increase HCC levels through cross-reactivity in an immunoassay. In general, topical or inhalation corticosteroids may also exert some systemic effects, and thereby decrease HCC. One study described that corticosteroid use, which was not further specified, increased HCC (64). LC-MS/MS is not limited by cross-reactivity, but false increases may still occur with synthetic corticosteroids that are structurally similar to cortisol.

\section{Future directions}

Scalp hair analysis is a promising and rapidly developing additional tool to evaluate HPA axis activity. One important practical advantage is that hair sampling can easily be performed in an outpatient setting, and is not dependent on patient adherence to sampling instructions (as with $24 \mathrm{~h}$ urine collections or LNSC) or medication intake (as in DST). Furthermore, the ability to create retrospective timelines creates the opportunity to investigate patterns of cortisol exposure for months up to years back in time, with the collection of a single hair sample. For HCC to become a fully established method in clinical practice, findings concerning Cushing's disease need to be replicated. It should furthermore be considered that causes of pseudo-CS such as alcohol use $(25,26,71)$, obesity (29) and depression (39) have also been associated with increased HCC.

In observational research, the association between increased HCC and an adverse cardiometabolic risk profile appears to be the most consistent clinical finding. All published studies which investigated this association thus far are cross-sectional, and therefore no conclusions about causal inferences can be made. Because an extreme glucocorticoid excess (i.e. CS) is strongly associated with metabolic syndrome components (central adiposity, insulin resistance, dyslipidemia and hypertension), an increased long-term cortisol exposure within the normal range is theoretically likely to have a causative role in increasing cardiovascular risk. However, the possibility of reverse causality cannot be discarded. For instance, chronic stress and increased psychopathology associated with obesity (72), or altered steroid metabolism could lead to increased cortisol levels. Therefore, there is a clear need for longitudinal studies examining the influence of long-term cortisol exposure on cardiovascular risk.

Measurement of hair cortisol creates an unique opportunity to study the effects of interventions on long-term cortisol due to the possibility of creating retrospective timelines by dividing hair samples into segments. Randomized controlled trials could easily incorporate this method, because depending on the length of the hair, a single hair sample may be used to evaluate changes in cortisol production over multiple months. Currently, several published examples of this type of study exist. In 18 participants who underwent a mindfulness or cognitive behavioral intervention for smoking cessation, Goldberg et al. (73) showed a significant decrease in HCC between the hair segments corresponding to the period before the intervention, and the period after the intervention. The decrease was present in both treatment arms, and since no placebo group was present, a conclusion about the cause of the observed decrease cannot be drawn. In contrast, in a randomized clinical trial involving 261 patients with structural heart disease, we observed no difference in between patient who underwent a mindfulness based stress reduction intervention, compared to patients who were assigned to the control group, although the whole study population on average showed a decrease in HCC during the study (32). Future studies will show if, and which behavioral and medical interventions are capable of modulating longterm cortisol exposure as measured with HCC. Evidence of observational studies indicate that a decrease may be associated with health benefits, especially in cardiometabolic health.

\section{Conclusions}

Analysis of cortisol in scalp hair has provided researchers with a readily applicable tool to measure cortisol exposure over extended periods of time. Studies in the past years indicate that hair cortisol may provide a new tool to investigate the HPA axis activity in clinical practice, such as in (cyclic) CS and hydrocortisone replacement therapy monitoring. Scalp hair offers of an easy collection procedure, and importantly, the addition of long-term retrospective information of cortisol exposure. Hair cortisol levels have been shown to be altered in association with a wide array of stressors and pathological conditions. Hair analysis has shown consistent associations between increased long-term cortisol and an adverse cardio metabolic risk profile, which may pave the way towards a new method for future cardiovascular risk stratification and evaluation of treatment.

Declaration of interest

The authors declare that there is no conflict of interest that could be perceived as prejudicing the impartiality of the review. 


\section{Funding}

The last author (E F C van Rossum) supported by the Thrasher Research Fund (TRF11643), The Netherlands Brain Foundation (grant number F2011(1)-12) and an Erasmus MC Fellowship.

\section{Acknowledgements}

The authors wish to thank Sabine M Staufenbiel for her help with literature review and reviewing our manuscript.

This paper forms part of a special issue of European Journal of Endocrinology on Cushing's syndrome. This article is adapted from work presented at the IMPROCUSH-1: Improving Outcome of Cushing's Syndrome symposium, 12-14 October 2014. The meeting was supported by the European Science Foundation, Deutsche Forschungsgemeinschaft, Carl Friedrich von Siemens Stiftung, European Neuroendocrine Association and the Deutsche Gesellschaft für Endokrinologie. The opinions or views expressed in this article are those of the authors, and do not necessarily reflect the opinions or recommendations of the European Science Foundation, Deutsche Forschungsgemeinschaft, Carl Friedrich von Siemens Stiftung, European Neuroendocrine Association and the Deutsche Gesellschaft für Endokrinologie

\section{References}

1 Quax RA, Manenschijn L, Koper JW, Hazes JM, Lamberts SWJ, van Rossum EFC \& Feelders RA. Glucocorticoid sensitivity in health and disease. Nature Reviews. Endocrinology 20139 670-686. (doi:10.1038/ nrendo.2013.183)

2 Nieman LK, Biller BM, Findling JW, Newell-Price J, Savage MO, Stewart PM \& Montori VM. The diagnosis of Cushing's syndrome: an Endocrine Society Clinical Practice Guideline. Journal of Clinical Endocrinology and Metabolism 200893 1526-1540. (doi:10.1210/ jc.2008-0125)

3 Tsigos C \& Chrousos GP. Hypothalamic-pituitary-adrenal axis, neuroendocrine factors and stress. Journal of Psychosomatic Research 200253 865-871. (doi:10.1016/S0022-3999(02)00429-4)

4 Cooper GA, Kronstrand R, Kintz P \& Society of Hair T . Society of Hair Testing guidelines for drug testing in hair. Forensic Science International 2012218 20-24. (doi:10.1016/j.forsciint.2011.10.024)

5 Sauve B, Koren G, Walsh G, Tokmakejian S \& Van Uum SH. Measurement of cortisol in human hair as a biomarker of systemic exposure. Clinical and Investigative Medicine. Médecine Clinique et Experimental 200730 E183-E191.

6 Kirschbaum C, Tietze A, Skoluda N \& Dettenborn L. Hair as a retrospective calendar of cortisol production - increased cortisol incorporation into hair in the third trimester of pregnancy. Psychoneuroendocrinology 200934 32-37. (doi:10.1016/j.psyneuen.2008.08.024)

7 Gao W, Stalder T, Foley P, Rauh M, Deng H \& Kirschbaum C. Quantitative analysis of steroid hormones in human hair using a column-switching LC-APCI-MS/MS assay. Journal of Chromatography. B, Analytical Technologies in the Biomedical and Life Sciences 2013928 1-8. (doi:10.1016/j.jchromb.2013.03.008)

8 D'Anna-Hernandez KL, Ross RG, Natvig CL \& Laudenslager ML. Hair cortisol levels as a retrospective marker of hypothalamic-pituitary axis activity throughout pregnancy: comparison to salivary cortisol. Physiology \& Behavior 2011104 348-353. (doi:10.1016/j.physbeh.2011. 02.041)

9 Manenschijn L, Koper JW, Lamberts SW \& van Rossum EF. Evaluation of a method to measure long term cortisol levels. Steroids $2011 \mathbf{7 6}$ 1032-1036. (doi:10.1016/j.steroids.2011.04.005)

10 Noppe G, de Rijke YB, Dorst K, van den Akker ELT \& van Rossum EFC. LC-MS/MS based method for long-term steroid profiling in human scalp hair. Clinical Endocrinology 2015 [In press]. (doi:10.1111/ cen.12781)

11 Staufenbiel SM, Penninx BW, Spijker AT, Elzinga BM \& van Rossum EF. Hair cortisol, stress exposure, and mental health in humans: a systematic review. Psychoneuroendocrinology 201338 1220-1235. (doi:10.1016/j.psyneuen.2012.11.015)

12 Wester VL, Lamberts SW \& van Rossum EF. Advances in the assessment of cortisol exposure and sensitivity. Current Opinion in Endocrinology, Diabetes, and Obesity 201421 306-311. (doi:10.1097/MED. 0000000000000077)

13 Russell E, Kirschbaum C, Laudenslager ML, Stalder T, de Rijke Y, van Rossum EF, Van Uum S \& Koren G. Toward standardization of hair cortisol measurement: results of the first international interlaboratory round robin. Therapeutic Drug Monitoring 201537 71-75. (doi:10.1097/ FTD.0000000000000148)

14 Manenschijn L, Koper JW, van den Akker EL, de Heide LJ, Geerdink EA, de Jong FH, Feelders RA \& van Rossum EF. A novel tool in the diagnosis and follow-up of (cyclic) Cushing's syndrome: measurement of longterm cortisol in scalp hair. Journal of Clinical Endocrinology and Metabolism 201297 E1836-E1843. (doi:10.1210/jc.2012-1852)

15 Thomson S, Koren G, Fraser LA, Rieder M, Friedman TC \& Van Uum SH. Hair analysis provides a historical record of cortisol levels in Cushing's syndrome. Experimental and Clinical Endocrinology \& Diabetes 2010118 133-138. (doi:10.1055/s-0029-1220771)

16 van Rossum EFC, Manenschijn L \& Feelders RA. Measuring cortisol levels in hair: potential clinical applications in Cushing's syndrome. Expert Review of Endocrinology \& Metabolism 20127 123-125. (doi:10. 1586/eem.11.95)

17 Friedman TC, Ghods DE, Shahinian HK, Zachery L, Shayesteh N, Seasholtz S, Zuckerbraun E, Lee ML \& McCutcheon IE. High prevalence of normal tests assessing hypercortisolism in subjects with mild and episodic Cushing's syndrome suggests that the paradigm for diagnosis and exclusion of Cushing's syndrome requires multiple testing. Hormone and Metabolic Research 201042 874-881. (doi:10.1055/s-00301263128)

18 Meinardi JR, Wolffenbuttel BH \& Dullaart RP. Cyclic Cushing's syndrome: a clinical challenge. European Journal of Endocrinology 2007 157 245-254. (doi:10.1530/EJE-07-0262)

19 Staufenbiel SM, Andela CD, Manenschijn L, Pereira AM, van Rossum EF $\&$ Biermasz NR. Increased hair cortisol concentrations and BMI in patients with pituitary-adrenal disease on hydrocortisone replacement. Journal of Clinical Endocrinology and Metabolism 2015 [In press]. (doi:10.1210/jc.2014-4328)

20 Gow R, Koren G, Rieder M \& Van Uum S. Hair cortisol content in patients with adrenal insufficiency on hydrocortisone replacement therapy. Clinical Endocrinology 201174 687-693. (doi:10.1111/j.13652265.2011.04001.x)

21 Noppe G, van Rossum EF, Vliegenthart J, Koper JW \& van den Akker EL. Elevated hair cortisol concentrations in children with adrenal insufficiency on hydrocortisone replacement therapy. Clinical Endocrinology 201481 820-825. (doi:10.1111/cen.12551)

22 Manenschijn L, Quinkler M \& van Rossum EF. Hair cortisol measurement in mitotane-treated adrenocortical cancer patients. Hormone and Metabolic Research 201446 299-304. (doi:10.1055/s-00341370961)

23 Kamps AW, Molenmaker M, Kemperman R, van der Veen BS, Bocca G \& Veeger NJ. Children with asthma have significantly lower long-term cortisol levels in their scalp hair than healthy children. Acta Paediatrica 2014103 957-961. (doi:10.1111/apa.12685)

24 Walker BR. Glucocorticoids and cardiovascular disease. European Journal of Endocrinology 2007157 545-559. (doi:10.1530/EJE-07-0455)

25 Manenschijn L, Schaap L, van Schoor NM, van der Pas S, Peeters GM, Lips P, Koper JW \& van Rossum EF. High long-term cortisol levels, measured in scalp hair, are associated with a history of cardiovascular disease. Journal of Clinical Endocrinology and Metabolism 201398 2078-2083. (doi:10.1210/jc.2012-3663) 
26 Feller S, Vigl M, Bergmann MM, Boeing H, Kirschbaum C \& Stalder T. Predictors of hair cortisol concentrations in older adults. Psychoneuroendocrinology 201439 132-140. (doi:10.1016/j.psyneuen.2013.10.007)

27 Pereg D, Gow R, Mosseri M, Lishner M, Rieder M, Van Uum S \& Koren G. Hair cortisol and the risk for acute myocardial infarction in adult men. Stress 2011 14 73-81. (doi:10.3109/10253890.2010.511352)

28 Stalder T, Kirschbaum C, Alexander N, Bornstein SR, Gao W, Miller R, Stark S, Bosch JA \& Fischer JE. Cortisol in hair and the metabolic syndrome. Journal of Clinical Endocrinology and Metabolism 201398 2573-2580. (doi:10.1210/jc.2013-1056)

29 Wester VL, Staufenbiel SM, Veldhorst MA, Visser JA, Manenschijn L, Koper JW, Klessens-Godfroy FJ, van den Akker EL \& van Rossum EF. Long-term cortisol levels measured in scalp hair of obese patients. Obesity 201422 1956-1958. (doi:10.1002/oby.20795)

30 Veldhorst MA, Noppe G, Jongejan MH, Kok CB, Mekic S, Koper JW, van Rossum EF \& van den Akker EL. Increased scalp hair cortisol concentrations in obese children. Journal of Clinical Endocrinology and Metabolism 201499 285-290. (doi:10.1210/jc.2013-2924)

31 Manenschijn L, van Kruysbergen RG, de Jong FH, Koper JW \& van Rossum EF. Shift work at young age is associated with elevated long-term cortisol levels and body mass index. Journal of Clinical Endocrinology and Metabolism 201196 E1862-E1865. (doi:10.1210/ jc.2011-1551)

32 Younge JO, Wester VL, van Rossum EF, Gotink RA, Wery MF, Utens EM, Hunink MG \& Roos-Hesselink JW. Cortisol levels in scalp hair of patients with structural heart disease. International Journal of Cardiology 2015184 71-78. (doi:10.1016/j.ijcard.2015.02.005)

33 Stalder T, Steudte S, Alexander N, Miller R, Gao W, Dettenborn L \& Kirschbaum C. Cortisol in hair, body mass index and stress-related measures. Biological Psychiatry 201290 218-223. (doi:10.1016/ j.biopsycho.2012.03.010)

34 Braig S, Grabher F, Ntomchukwu C, Reister F, Stalder T, Kirschbaum C, Genuneit J \& Rothenbacher D. Determinants of maternal hair cortisol concentrations at delivery reflecting the last trimester of pregnancy. Psychoneuroendocrinology 201552 289-296. (doi:10.1016/j.psyneuen. 2014.12.006)

35 Pereg D, Chan J, Russell E, Berlin T, Mosseri M, Seabrook JA, Koren G \& Van Uum S. Cortisol and testosterone in hair as biological markers of systolic heart failure. Psychoneuroendocrinology 201338 2875-2882. (doi:10.1016/j.psyneuen.2013.07.015)

36 Burke HM, Davis MC, Otte C \& Mohr DC. Depression and cortisol responses to psychological stress: a meta-analysis. Psychoneuroendocrinology 200530 846-856. (doi:10.1016/j.psyneuen.2005.02.010)

37 Vreeburg SA, Hoogendijk WJ, van Pelt J, Derijk RH, Verhagen JC, van Dyck R, Smit JH, Zitman FG \& Penninx BW. Major depressive disorder and hypothalamic-pituitary-adrenal axis activity: results from a large cohort study. Archives of General Psychiatry 200966 617-626. (doi:10.1001/archgenpsychiatry.2009.50)

38 Holsboer F. The corticosteroid receptor hypothesis of depression. Neuropsychopharmacology 200023 477-501. (doi:10.1016/S0893133X(00)00159-7)

39 Dettenborn L, Muhtz C, Skoluda N, Stalder T, Steudte S, Hinkelmann K, Kirschbaum C \& Otte C. Introducing a novel method to assess cumulative steroid concentrations: increased hair cortisol concentrations over 6 months in medicated patients with depression. Stress 201215 348-353. (doi:10.3109/10253890.2012.654479)

40 Manenschijn L, Spijker AT, Koper JW, Jetten AM, Giltay EJ, Haffmans J, Hoencamp E \& van Rossum EF. Long-term cortisol in bipolar disorder: associations with age of onset and psychiatric co-morbidity. Psychoneuroendocrinology 201237 1960-1968. (doi:10.1016/j.psyneuen.2012. 04.010)

41 Steudte S, Stalder T, Dettenborn L, Klumbies E, Foley P, Beesdo-Baum K $\&$ Kirschbaum C. Decreased hair cortisol concentrations in generalised anxiety disorder. Psychiatry Research 2011186 310-314. (doi:10.1016/j. psychres.2010.09.002)
42 Meewisse ML, Reitsma JB, de Vries GJ, Gersons BP \& Olff M. Cortisol and post-traumatic stress disorder in adults: systematic review and meta-analysis. British Journal of Psychiatry 2007191 387-392. (doi:10.1192/bjp.bp.106.024877)

43 Steudte S, Kirschbaum C, Gao W, Alexander N, Schonfeld S, Hoyer J \& Stalder T. Hair cortisol as a biomarker of traumatization in healthy individuals and posttraumatic stress disorder patients. Biological Psychiatry 201374 639-646. (doi:10.1016/j.biopsych.2013.03.011)

44 Luo H, Hu X, Liu X, Ma X, Guo W, Qiu C, Wang Y, Wang Q, Zhang X, Zhang $\mathrm{W}$ et al. Hair cortisol level as a biomarker for altered hypothalamic-pituitary-adrenal activity in female adolescents with posttraumatic stress disorder after the 2008 Wenchuan earthquake. Biological Psychiatry 201272 65-69. (doi:10.1016/j.biopsych.2011. 12.020)

45 Wang L, Cao C, Wang W, Xu H, Zhang J, Deng H \& Zhang X. Linking hair cortisol levels to phenotypic heterogeneity of posttraumatic stress symptoms in highly traumatized Chinese women. Biological Psychiatry 201577 e21-e22. (doi:10.1016/j.biopsych.2014.05.026)

46 Steudte S, Kolassa IT, Stalder T, Pfeiffer A, Kirschbaum C \& Elbert T. Increased cortisol concentrations in hair of severely traumatized Ugandan individuals with PTSD. Psychoneuroendocrinology 201136 1193-1200. (doi:10.1016/j.psyneuen.2011.02.012)

47 Hellhammer DH, Wust S \& Kudielka BM. Salivary cortisol as a biomarker in stress research. Psychoneuroendocrinology 200934 163-171. (doi:10.1016/j.psyneuen.2008.10.026)

48 Skoluda N, Dettenborn L, Stalder T \& Kirschbaum C. Elevated hair cortisol concentrations in endurance athletes. Psychoneuroendocrinology 201237 611-617. (doi:10.1016/j.psyneuen.2011.09.001)

49 Van Uum SH, Sauve B, Fraser LA, Morley-Forster P, Paul TL \& Koren G. Elevated content of cortisol in hair of patients with severe chronic pain: a novel biomarker for stress. Stress 200811 483-488. (doi:10.1080/ 10253890801887388)

50 Kalra S, Einarson A, Karaskov T, Van Uum S \& Koren G. The relationship between stress and hair cortisol in healthy pregnant women. Clinical and Investigative Medicine. Médecine Clinique et Experimental 200730 E103-E107.

51 Karlen J, Ludvigsson J, Frostell A, Theodorsson E \& Faresjo T. Cortisol in hair measured in young adults - a biomarker of major life stressors? BMC Clinical Pathology 201111 12. (doi:10.1186/1472-6890-11-12)

52 Dowlati Y, Herrmann N, Swardfager W, Thomson S, Oh PI, Van Uum S, Koren G \& Lanctot KL. Relationship between hair cortisol concentrations and depressive symptoms in patients with coronary artery disease. Neuropsychiatric Disease and Treatment 20106 393-400

53 Vanaelst B, Michels N, De Vriendt T, Huybrechts I, Vyncke K, Sioen I, Bammann K, Rivet N, Raul JS, Molnar D et al. Cortisone in hair of elementary school girls and its relationship with childhood stress. European Journal of Pediatrics 2013172 843-846. (doi:10.1007/s00431013-1955-1)

54 Staufenbiel SM, Koenders MA, Giltay EJ, Elzinga BM, Manenschijn L, Hoencamp E, van Rossum EF \& Spijker AT. Recent negative life events increase hair cortisol concentrations in patients with bipolar disorder. Stress 201417 451-459. (doi:10.3109/10253890.2014.968549)

55 Grassi-Oliveira R, Pezzi JC, Daruy-Filho L, Viola TW, Francke ID, Leite CE \& Brietzke E. Hair cortisol and stressful life events retrospective assessment in crack cocaine users. American Journal of Drug and Alcohol Abuse 201238 535-538. (doi:10.3109/00952990.2012.694538)

56 Groeneveld MG, Vermeer HJ, Linting M, Noppe G, van Rossum EF \& van IJzendoorn MH. Children's hair cortisol as a biomarker of stress at school entry. Stress 201316 711-715. (doi:10.3109/10253890.2013. 817553)

57 Stalder T, Tietze A, Steudte S, Alexander N, Dettenborn L \& Kirschbaum C. Elevated hair cortisol levels in chronically stressed dementia caregivers. Psychoneuroendocrinology 201447 26-30. (doi:10.1016/j.psyneuen.2014.04.021)

58 Steinisch M, Yusuf R, Li J, Stalder T, Bosch JA, Rahman O, Strumpell C, Ashraf H, Fischer JE \& Loerbroks A. Work stress and hair cortisol levels 
among workers in a Bangladeshi ready-made garment factory - results from a cross-sectional study. Psychoneuroendocrinology 2014 50C 20-27. (doi:10.1016/j.psyneuen.2014.08.001)

59 Dettenborn L, Tietze A, Bruckner F \& Kirschbaum C. Higher cortisol content in hair among long-term unemployed individuals compared to controls. Psychoneuroendocrinology 201035 1404-1409. (doi:10.1016/ j.psyneuen.2010.04.006)

60 Noppe G, Van Rossum EF, Koper JW, Manenschijn L, Bruining GJ, de Rijke YB \& van den Akker EL. Validation and reference ranges of hair cortisol measurement in healthy children. Hormone Research in Paediatrics 201482 97-102. (doi:10.1159/000362519)

61 Dettenborn L, Tietze A, Kirschbaum C \& Stalder T. The assessment of cortisol in human hair: associations with sociodemographic variables and potential confounders. Stress 201215 578-588. (doi:10.3109/ 10253890.2012.654479)

62 Schütz H, Ahrens B, Erdmann F \& Rochholz G. Nachweis von Arzneiund anderen Fremdstoffen in Haaren. Pharmazie in unserer Zeit 199322 65-78.

63 Loussouarn G, El Rawadi C \& Genain G. Diversity of hair growth profiles. International Journal of Dermatology 200544 (Suppl 1) 6-9. (doi:10.1111/j.1365-4632.2005.02800.x)

64 Wells S, Tremblay PF, Flynn A, Russell E, Kennedy J, Rehm J, Van Uum S, Koren G \& Graham K. Associations of hair cortisol concentration with self-reported measures of stress and mental health-related factors in a pooled database of diverse community samples. Stress 201417 334-342. (doi:10.3109/10253890.2014.930432)

65 Boesch M, Sefidan S, Annen H, Ehlert U, Roos L, Van Uum S, Russell E, Koren G \& La Marca R. Hair cortisol concentration is unaffected by basic military training, but related to sociodemographic and environmental factors. Stress 201518 35-41. (doi:10.3109/10253890.2014. 974028)
66 Gao W, Xie Q, Jin J, Qiao T, Wang H, Chen L, Deng H \& Lu Z. HPLC-FLU detection of cortisol distribution in human hair. Clinical Biochemistry 201043 677-682. (doi:10.1016/j.clinbiochem.2010.01.014)

67 Stalder T, Steudte S, Miller R, Skoluda N, Dettenborn L \& Kirschbaum C. Intraindividual stability of hair cortisol concentrations. Psychoneuroendocrinology 201237 602-610. (doi:10.1016/j.psyneuen.2011.08.007)

68 Russell E, Koren G, Rieder M \& Van Uum SH. The detection of cortisol in human sweat: implications for measurement of cortisol in hair. Therapeutic Drug Monitoring 201436 30-34. (doi:10.1097/FTD. Ob013e31829daa0a)

69 Grass J, Kirschbaum C, Miller R, Gao W, Steudte-Schmiedgen S \& Stalder T. Sweat-inducing physiological challenges do not result in acute changes in hair cortisol concentrations. Psychoneuroendocrinology 2015 53C 108-116. (doi:10.1016/j.psyneuen.2014.12.023)

70 Tiganescu A, Walker EA, Hardy RS, Mayes AE \& Stewart PM. Localization, age- and site-dependent expression, and regulation of $11 \beta$-hydroxysteroid dehydrogenase type 1 in skin. Journal of Investigative Dermatology 2011131 30-36. (doi:10.1038/jid.2010.257)

71 Stalder T, Kirschbaum C, Heinze K, Steudte S, Foley P, Tietze A \& Dettenborn L. Use of hair cortisol analysis to detect hypercortisolism during active drinking phases in alcohol-dependent individuals. Biological Psychiatry 201085 357-360. (doi:10.1016/j.biopsycho.2010. 08.005)

72 Ells LJ, Lang R, Shield JP, Wilkinson JR, Lidstone JS, Coulton S \& Summerbell CD. Obesity and disability - a short review. Obesity Reviews 20067 341-345. (doi:10.1111/j.1467-789X.2006.00233.x)

73 Goldberg SB, Manley AR, Smith SS, Greeson JM, Russell E, Van Uum S, Koren G \& Davis JM. Hair cortisol as a biomarker of stress in mindfulness training for smokers. Journal of Alternative and Complementary Medicine 201420 630-634. (doi:10.1089/acm. 2014.0080)

Received 20 March 2015

Revised version received 22 April 2015

Accepted 28 April 2015 